\title{
Performance analysis of adaptive OFDM modulation scheme in VLC vehicular communication network in realistic noise environment
}

\author{
Hasan Farahneh* (D), Fatima Hussain and Xavier Fernando
}

\begin{abstract}
Optical wireless communications (OWC) has emerged as a strong candidate for wireless communications, due to the capacity limitation in the radio frequency (RF) spectrum. Especially visible light communication (VLC) has great potential for short-range outdoor vehicular communications, as vehicle LED lights also transmit data. However, outdoor VLC channels vary fast and, experience multipath scattering and reflection resulting in time domain dispersion. Outdoor VLC links are also subjected to high levels of ambient noise, especially from the sun. Orthogonal frequency-division multiplexing (OFDM), which has proven robustness to multi path fading and noise effects in RF links can also be deployed in VLC links. In this paper, optical OFDM (O-OFDM) along with adaptive modulation scheme is investigated in VLC for vehicle to vehicle $($ V2V) communications. A $(2 \times 2)$ multiple input multiple output (MIMO) channel, with multiple polarimetric bidirectional reflections and realistic sunlight interference is considered. Two schemes of O-OFDM; direct current biased optical OFDM (DCO-OFDM) and asymmetrically clipped optical OFDM (ACO-OFDM) are investigated. Simulation results of the proposed model show increase in data rates up to $50 \mathrm{Mbps}$ along with reduced bit error rate (BER) under both line of sight (LOS) and non-LOS and high noise conditions.
\end{abstract}

Keywords: MIMO, VLC, V2V, Optical OFDM, Adaptive modulation, Bit loading

\section{Introduction}

Visible light communication (VLC) is emerging as an attractive alternative to radio frequency $(\mathrm{RF})$ wireless communications [1]. VLC-based vehicle to vehicle (V2V) and vehicle to infrastructure (V2I) communications systems have gained greater attention, primarily due to the fast development in high-intensity light emitting diode (LED) devices in vehicles that has the ability to switch fast and transmit data in addition to lighting. Vehicles are increasingly equipped with image/video sensors that may also function as optical receivers with some modification. Since, the VLC transmitters and receivers can be easily added to modern vehicles, the VLC-based approach promises a new episode in cost-effective V2V and V2I

*Correspondence: hfarahne@Ryerson.ca

Department of Electrical and Computer Engineering, Ryerson University, 350 Victoria Street, M5B 2K3 Toronto, Canada communications, especially with the widespread deployment of autonomous vehicles and intelligent transportation systems (ITS), that improve road safety and provide better traffic flow [2].

Although, the optical spectrum has abundant bandwidth, the VLC channels have inherent scattering and reflection properties and undergo dispersion due to multipath dispersion. This results in inter-symbol interference (ISI) that leads to low data rate. Most previous work on optical wireless communications consider indoor environments, which is quasi stationary and well confined. However, the outdoor environment is very different. It is characterized by short channel coherent time with moving vehicles, high reflection due to shiny vehicle bodies and, high ambient noise.

Many approaches are taken to mitigate these impairments. Time domain equalization (TDE) techniques are prevalent in single carrier systems. Code division multiplexing (CDMA) and adaptive de-noising are also 
tried [35]. However, orthogonal frequency-division multiplexing (OFDM) approach, which is an advanced multicarrier technique can be very effective in VLC links [2]. OFDM has been proven successful in many RF wireless (e.g., IEEE 802.11, LTE, WiMAX) and wire-line standards (e.g., DSL) [3]. Optical OFDM (O-OFDM) schemes promised to have better optical power efficiency, reduced ISI, and very low bit error rate (BER) compared to conventional optical modulation schemes; such as on-off-keying (OOK) and pulse position modulation (PPM) [4]. OFDM also enables adaptive modulation depths widely referred to as 'bit loading.'

Note that the OFDM is originally developed for bipolar electrical communication systems that has both positive and negative signal components. In order to make it suitable for the unipolar VLC environment, there are few techniques such as direct current biased optical OFDM (DCO-OFDM) and asymmetrically clipped optical OFDM (ACO-OFDM) are investigated in the literature.

Multiple input, multiple output (MIMO) systems have gained considerable attention in VLC networks due to their high data rate capabilities over longer distances. In addition to overcoming the multipath fading and interference effects, the MIMO approach can be very effective to mitigate the shadowing effects which are severe in the optical realm due to strict line of sight properties [11]. Therefore, the combination of OFDM and MIMO can be a powerful physical layer solution for high-speed vehicular VLC systems for high bandwidth applications [5]. Our literature survey shows, until now MIMO and OFDM combination has been mostly investigated with non-adaptive modulation schemes except for few existing works that have studied adaptive OFDM in indoor applications [6]. Furthermore, usually researchers have assumed Gaussian channels. However, we have assumed a more realistic polarimetric bidirectional reflective channel model with adaptive modulation.

\subsection{Relevant literature}

There has been little work done in outdoor VLC applications. Therefore, there is a need of extensive research efforts in this domain. There is also very little work done on incorporating OFDM and VLC. Performance of VLC system using space time block coded OFDM (STBC-OFDM) technique for ITS was investigated in [7]. Authors in [8] presented single input single output V2VVLC system with a DCO-OFDM. In [9], BER, and effect of receiver height were investigated for car-to-car VLC system using $2 \times 2$ MIMO. Authors in [10] characterized the channel impulse response of VLC-based V2V communication system using MIMO model. The effect of the shadowing on the VLC-based V2V system was investigated in [11]. In [12], authors presented a V2V communication system by using LED as a transmitter and a camera as a receiver. Authors in [13], introduced an environment-adaptive VLC receiver for a vehicular communication system. Their proposed receiver can support long-distance communications even during dynamic and unfriendly traffic and atmospheric conditions. In [14], authors suggested DCO-OFDM-based MIMO transmission scheme for vehicular communication and evaluated the performances of various MIMO modes; such as spatial multiplexing, repetition code with different modulation techniques, and various transmitter-receiver selection. However, it was observed that spatial multiplexing technique suffers from channel correlation, while repetition code is unsuitable for high modulation orders.

\subsection{Sunlight effect on outdoor VLC systems}

It is a well-known fact that $47 \%$ of sunlight irradiance falls within the visible light band of the frequency spectrum [15]. The meteorological conditions of various network locations determine the level of sunlight. The major challenge of an outdoor VLC system is the strong influence of the ambient (mainly sun) light. Daylight contributes to unmodulated signal being received along with desired transmitted signal. This noise signal constitutes more average power than the desired signal during daylight even with strong optical filtering. Therefore, natural light incident on the receiver generates large amount of shot noise that affects the system performance. The signal to noise ratio can be very low or even negative.

Most of the previous work in outdoor VLC system did not consider the effect of sunlight noise nor its fluctuations at different times of the year at different locations. Only few researches have considered the effects of sunlight on outdoor VLC system. In [16], authors presented a daylight noise model based on Blackbody radiations, to capture the effect of ambient-light noise. In [15], authors considered the impact of sunlight as a function of location, time, and for various reflecting surfaces. In [17], authors presented analytical analysis by investigating effects of sunlight irradiance on VLC system, in terms of data rate and BER degradation.

Note that sunlight noise is non-stationary and very high in magnitude [36]. Different means of noise cancellation, such as wavelet-based signal processing approach and differential receiver-based hardware approach, has been proposed earlier [37]. However, due to the complex nature of the process, real-time de-noising has not been very fruitful.

From the foregoing, outdoor VLC technology is in its infancy and requires further research efforts in several areas, including channel modeling, physical layer design, and upper-layer protocols. That is the objective of this paper.

In this work, two O-OFDM approaches are considered in the presence of sunlight and reflectors. To the 
best of the authors' knowledge, this is the first time a practical outdoor VLC channel with real sunlight noise, clipping noise, and multiple reflections is considered with O-OFDM modulation.

The main contributions of this work are

- A $(2 \times 2)$ MIMO scenario for inter-vehicular communication.

- An adaptive modulation scheme incorporating a DCO-OFDM and an ACO-OFDM schemes for VLC-based V2V system.

- Bit-loading algorithm for performance improvement and singular value decomposition (SVD) technique for enhanced optimization.

- Performance analysis of the outdoor VLC environment with realistic sun light noise.

The rest of the paper is organized as follows. In Section 2, the methodology and system consideration are presented. In Section 3, a $2 \times 2$ optical MIMO model for VLC-based V2V system is presented. Section 4 describes DCO-OFDM and ACO-OFDM optical OFDM schemes. Also, comparison between both schemes is performed considering clipping errors and spectral efficiencies. In Section 5, analysis of different types of noises in the proposed system is performed. Analytical calculations and performance analysis along with bit loading algorithm is presented in Section 6. Simulation results and discussions are included in Section 7. Finally, conclusions are drawn in Section 8.

\section{Methodology}

MATLAB $^{\mathrm{TM}}$ software and Windows 10 desktop system are used to model the proposed system. The parameters and their values used in simulations are summarized in Tables 1, 2, and 3. Multipath propagation with one reflection is considered. All the reflectors surfaces are assumed to have the same reflective factor. Moreover, summer season with the clear sky is considered. It is assumed that the vehicles are moving at same speed in the same direction. The city of Toronto is considered for sunlight intensity calculations. We do Monte Carlo simulation for 5000 iterations for simulating the proposed VLC channel. White light XM-L LED for the vehicle's headlight is considered as a transmitter, and Hamamatsu-S8664 Avalanche Phot Diode (APD) is considered as a receiver.

\section{3 (2 × 2) Optical MIMO model for VLC-based V2V syetm}

The system model is presented in this section as shown in Fig. 1. The proposed VLC-based V2V system is comprised of two vehicles communicating with each other using, headlights and optical receivers equipped in the taillights. Headlights of first vehicle act as a transmitter. Since each
Table 1 Geometry parameters and its simulation values

\begin{tabular}{lll}
\hline Symbol & Description & Value \\
\hline$T, R, q$ & Transmitter, receiver, and reflector & $(1-2),(0-6)$ \\
$\phi_{R T}$ & Emitting angle from $T$ to $R$ & $30^{\circ}$ \\
$\theta_{R T}$ & Incident angle from $T$ to $R$ & $45^{\circ}$ \\
$R_{R T}$ & Distance between $T$ and $R$ & $20-120 \mathrm{~m}$ \\
$A_{R}$ & Effective area of $R$ & $1 \times 10^{-} 4 \mathrm{~m}^{2}$ \\
$T_{0}\left(\theta_{R T}\right)$ & Transmission coefficient of filter & 1 \\
$\Psi_{R T}$ & FOV of $R$ with respect to $T$ & $75^{\circ}$ \\
$g\left(\theta_{R T}\right)$ & The concentrator gain & 5 \\
$\phi_{q T}$ & Emitting angle between $T$ and $q$ & $30^{\circ}$ \\
$\theta_{q T}$ & Incident angle between $T$ and $q$ & $25^{\circ}$ \\
$A_{q}$ & Reflector area & $150 \mathrm{~cm}{ }^{2}$ \\
$\rho_{q}$ & The reflectivity & $0.8[20]$ \\
$\phi_{R q}$ & Emitting angle between $R$ and $q$ & $30^{\circ}$ \\
$\theta_{R q}$ & Incident angle from $q$ to $R$ & $45^{\circ}$ \\
$R_{q T}$ & Distance between $T$ and $q$ & $20-80 \mathrm{~m}$ \\
$R_{R q}$ & Distance between $q$ and $R$ & $20-80 \mathrm{~m}$ \\
$\Psi_{R q}$ & FoV of $R$ with respect to $q$ & $60^{\circ}$ \\
$h i$ & Height of the APD at the receiver & $70 \mathrm{~cm}$ \\
$I_{T}\left(\alpha_{T}, \beta_{T}\right)$ & Luminous intensity of $T$ & $1040 \mathrm{~cd}$ \\
$\left(\alpha, \beta_{T}\right)$ & Position angles of $T$ & $10^{\circ}, 10^{\circ}$ \\
\hline & &
\end{tabular}

car has two headlights and two taillights, a $2 \times 2 \mathrm{MIMO}$ link can be considered.

\subsection{Proposed system considerations}

LEDs at transmitter and avalanche photodiode (APDs) at receiver are considered. The market-weighted headlighting pattern source for the transmitter and receiver

Table 2 Noise parameters and its simulation values

\begin{tabular}{lll}
\hline Symbol & Description & Value \\
\hline APD model & (Si) Hamamatsu & $58664-1010$ \\
$\lambda(n \mathrm{~nm})$ & Visible light wave length & {$[350-750]$} \\
$G$ & APD gain & 50 \\
$P_{S}(\lambda)(/ x)$ & Sunlight intensity & 12000 \\
$q_{e}(C)$ & Electron charge & $1.9 \times 10^{-19}$ \\
$\theta_{\text {sun }}$ & Sunlight incident angle & $25^{\circ}$ \\
$B(\mathrm{MHz})$ & APD bandwidth & 65 \\
$I_{\mathrm{ds}}(n A)$ & Surface dark current & 10 \\
$I_{\mathrm{dg}}(\mu \mathrm{A}$ & Bulk dark current & 5100 \\
$K_{B}(J / K)$ & Boltzmann's constant & $1.38 \times 10^{-23}$ \\
$T_{k}(K)$ & Temperature & 298 \\
$F$ & APD noise figure & 0.2 \\
$\gamma(A / W)$ & Photodetector responsivity & 0.28 \\
$R_{L}$ in $\Omega$ & Load resistance & 50 \\
\hline
\end{tabular}


Table 3 Simulation parameters

\begin{tabular}{ll}
\hline The parameter & Value \\
\hline Toronto location & $43.6532^{\circ} \mathrm{N}, 79.3832^{\circ} \mathrm{W}$ \\
Weather & Clear \\
Number of FFT points & 64 \\
Subcarrier modulation scheme & $4-\mathrm{QAM}, 16-\mathrm{QAM}, . .256-\mathrm{QAM}$ \\
Total system bandwidth & $50 \mathrm{MHz}$ \\
Cyclic prefix length CP & 8 \\
Targeted BER & $10^{-5}$ \\
White LED XM-L & $1040(\mathrm{~cd})$ and LER $=100(\mathrm{Im} / \mathrm{W})$ \\
LED dynamic range $(V)$ & $V_{\text {ton }}=2.66, V_{\max }=3.25$ \\
Biased voltage $(V) b_{\mathrm{dc}}$ & $5 \mathrm{~V}$ \\
Vehicles speed $($ same direction) & $30 \mathrm{Km} / \mathrm{h}$ \\
\hline
\end{tabular}

[19] are considered ${ }^{1}$. This type of lights not only provide good road illumination, but also do not cause much glare to other commuters [21].

The outdoor VLC channel consists of parked and passing vehicles acting as reflectors ${ }^{2}$. Reflectance characteristics of a reflector surface depends on its nature and physical status, and changes with weather conditions. Therefore, a polarimetric bidirectional reflectance distribution function (BRDF) is used to model the reflectors in this work. The BRDF model can effectively model the polarimetric signatures of object surface; such as, paints or metal [22]. BRDF can be defined as [22]:

$$
f\left(\theta_{i}, \phi_{i}, \theta_{r}, \phi_{r}, \lambda\right)=\frac{d L_{r}\left(\theta_{r}, \phi_{r}\right)}{d E\left(\theta_{i}, \phi_{i}\right)}
$$

where $\theta_{i}$ and $\phi_{i}$ are incident polar and azimuth angles respectively, $\theta_{r}$ and $\phi_{r}$ are reflected polar and azimuth angles respectively, $\lambda$ is the wavelength, $L_{r}$ is the radiance scattering from the surface, and $E$ is the irradiance incident onto the surface.
The fast fading outdoor channel model given in [10] is used for the VLC channel. The line of sight (LOS) and non-line of sight (NLOS) paths are considered as shown in Figs. 1 and 2, respectively. The Cyclic-prefix (CP) of the OFDM scheme is calculated by considering ratio of the $\mathrm{CP}$ time to the useful time, fast Fourier transform (FFT) length, and the root mean squared (RMS) delay spread. Also, high ambient noise is considered for the system. Figure 2 demonstrates ray diagram and geometry of the proposed model and Table 1 list various symbols and their simulated values. While the noise parameters and their values are listed in Table 2.

\subsection{System analysis}

Considering Fig. 1, the received signal for $2 \times 2$ MIMO system is given as

$$
\mathbf{y}=\mathbf{H s}+\mathbf{z}
$$

where, $\mathbf{s}$ and $\mathbf{y}$ are transmitted and received signal vectors respectively, $\mathbf{H}$ indicates the channel matrix comprising of LOS, and NLOS (diffuse) components. Here, $\mathbf{z}$ is the total noise vector with variance $\sigma_{z}^{2}$ given as

$$
\sigma_{z}^{2}=\sigma_{n}^{2}+\sigma^{2} \text { ISI }+\sigma_{c}^{2}
$$

where $\sigma_{n}^{2}$ is the sum of the shot noise, dark noise, and thermal noise, $\sigma^{2}$ ISI is the ISI noise, $\sigma^{2}{ }_{c}$ is the clipping noise. The different components of (3) will be discussed in details in Section 4.

For $2 \times 2$ MIMO, the channel matrix $\mathbf{H}$ is given as

$$
\mathbf{H}=\left[\begin{array}{ll}
h_{11} & h_{12} \\
h_{21} & h_{22}
\end{array}\right]
$$

Each entry of matrix $\mathbf{H}$ can be given as

$$
h_{R T}(t)=h_{\mathrm{LOS}}(t)+h_{\mathrm{NLOS}}(t)
$$

where $h_{\mathrm{LOS}}(t)$ is the channel impulse response (CIR) for LOS path, and $h_{\mathrm{NLOS}}(t)$ is the CIR of NLOS path.

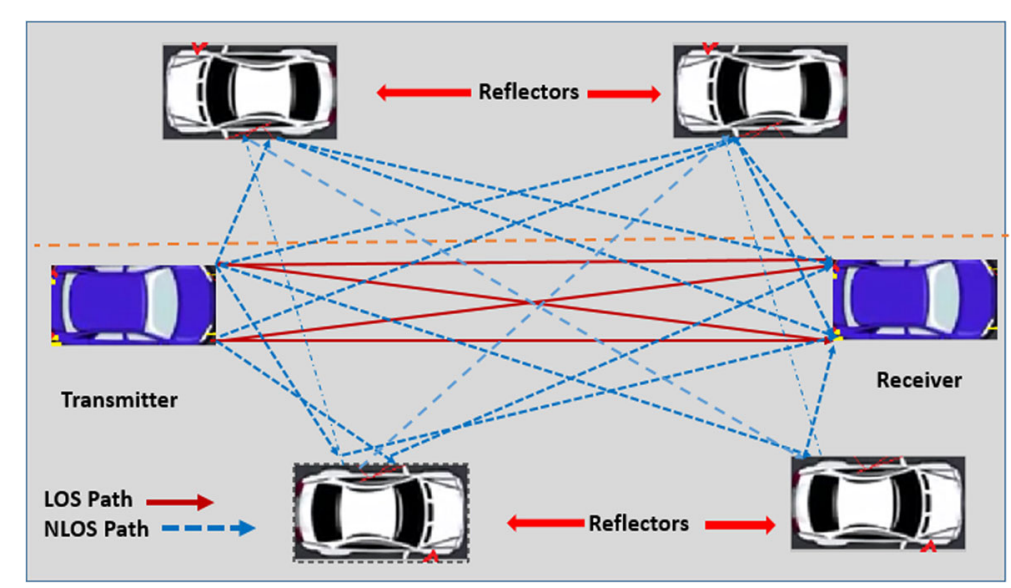

Fig. $1(2 \times 2)$ MIMO model for VLC-based system 


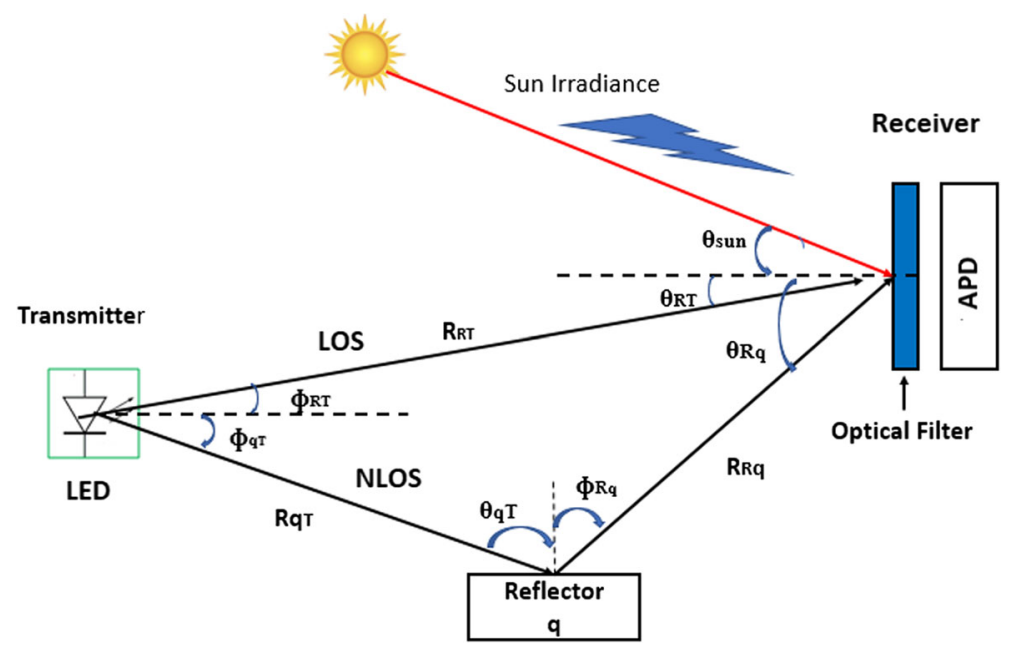

Fig. 2 Geometry of transmitter, receiver, and reflectors

For the LOS paths, CIR between transmitter $T$ and receiver $R, h_{R T_{\mathrm{LOS}}}(t)$ is given as

$$
\begin{aligned}
& h_{R T_{\mathrm{LOS}}}(t)= \\
& \begin{cases}\left(\sum_{R=1}^{2} \sum_{T=1}^{2} \frac{1}{\pi R_{R T}^{2}} \cos \left(\phi_{R T}\right) \cos \left(\theta_{R T}\right)\right. & \\
\left.A_{R} T_{o}\left(\theta_{R T}\right) g\left(\theta_{R T}\right) \delta\left(t-\frac{R_{R T}}{c}\right)\right) & \text { if } 0 \leq \theta_{R T} \leq \Psi_{R T} \\
0 & \text { if } \theta_{R T}>\Psi_{R T}\end{cases}
\end{aligned}
$$

where $\phi_{R T}$ denotes the emitting angle, $\theta_{R T}$ denotes the incident angle from source $T$ to receiver $R$ respectively, $R_{R T}$ denotes the distance between source $T$ and receiver $R, A_{R}$ denotes the area of the receiver $R, T_{o}\left(\theta_{R T}\right)$ denotes the signal transmission coefficient of an optical filter, $c$ denotes the speed of light, $\delta()$ denotes the impulse function, $\Psi_{\mathrm{RT}}$ denotes the field of view of the receiver $R$ with respect to the transmitter $T, g\left(\theta_{R T}\right)$ denotes the concentrator gain, $T$, and $R=1,2$.

Similarly, the NLOS impulse response $h_{R T_{\mathrm{NLOS}}}(t)$ is given as

$$
\begin{aligned}
h_{R T_{\mathrm{NLOS}}}(t) & = \begin{cases}\left(\sum_{R=1}^{2} \sum_{T=1}^{2} \sum_{q=0}^{Q} \frac{1}{\pi^{2} R_{q T}^{2} \cdot R_{R q}^{2}}\right. \\
\cos \left(\phi_{q T}\right) \cos \left(\theta_{q T}\right) A_{q} \rho_{q} \cos \left(\phi_{R q}\right) \cos \left(\theta_{R q}\right) . \\
\left.A_{R} T_{o}\left(\theta_{R q}\right) g\left(\theta_{R q}\right) \delta\left(t-\frac{R_{q T}+R_{R q}}{c}\right)\right) & \text { if } 0 \leq \theta_{R q} \leq \Psi_{R q} \\
0, & \text { if } \theta_{R q}>\Psi_{R q}\end{cases}
\end{aligned}
$$

where $\phi_{q T}$ denotes the emitting angle, $\theta_{q T}$ denotes the incident angle from source $T$ to the reflector $q$, respectively, $A_{q}$ denotes the $q^{t h}$ reflector's area, $\rho_{q}$ denotes the reflectivity, $\phi_{R q}$ denotes the emitting angle, $\theta_{R q}$ denotes the incident angle from reflector $q$ to the receiver $R$, respectively, $R_{q T}$ denotes the distance between transmitter $T$ and reflector $q, R_{R q}$ denotes the distance between reflector $q$ and the receiver $R, \Psi_{R q}$ denotes the field of view of the receiver $R$ with respect to the reflector $q$, $Q$ indicates the total number of reflectors.

The received optical power from direct paths is given as

$P_{r}^{\mathrm{LOS}}= \begin{cases}\left(\sum_{R=1}^{2} \sum_{T=1}^{2} \frac{I_{T}\left(\alpha_{T}, \beta_{T}\right) A_{R}}{(\operatorname{LER}) R_{R T}^{2}} \cos \left(\theta_{R T}\right)\right) & \text { if } 0 \leq \theta_{R T} \leq \Psi_{R T} \\ 0, & \text { if } \theta_{R T}>\Psi_{R T}\end{cases}$

where $I_{T}\left(\alpha_{T}, \beta_{T}\right)$ is the luminous intensity of the source $T$ from the direction $\left(\alpha_{T}, \beta_{T}\right)$ and its measured in candela (cd), $\alpha_{T}, \beta_{T}$ are the horizontal and vertical angles of the headlight respectively, and LER is the luminous efficacy of radiation of the LED.

The received optical power from reflected paths is given as

$P_{r}^{\mathrm{NLOS}}= \begin{cases}\left(\sum_{R=1}^{2} \sum_{T=1}^{2} \sum_{q=0}^{Q} \frac{I\left(\alpha_{T}, \beta_{T}\right) \sin \left(\theta_{q T}\right) A_{q} A_{R}}{(\operatorname{LER}) R_{q T}^{2}\left(R_{R q}^{2}+h i^{2}\right)}\right. & \\ \left.\rho_{q} \cos \left(\phi_{R q}\right) \cos \left(\theta_{R q}\right)\right) & \text { if } 0 \leq \theta_{R q} \leq \Psi_{R q} \\ 0 & \text { if } \theta_{R q}>\Psi_{R q}\end{cases}$

where $h_{i}$ is the hight of the receiver.

\section{Optical OFDM scheme of VLC-based V2V system}

This section discusses the proposed OFDM scheme for VLC-based V2V channel. In this work, the model presented in [18], and [10] is modified to include an O-OFDM with an adaptive modulation scheme. It helps to reduce ISI and also increases the data rate. Figure 3 shows the block diagram of the proposed optical OFDM scheme for VLC-based V2V system. 


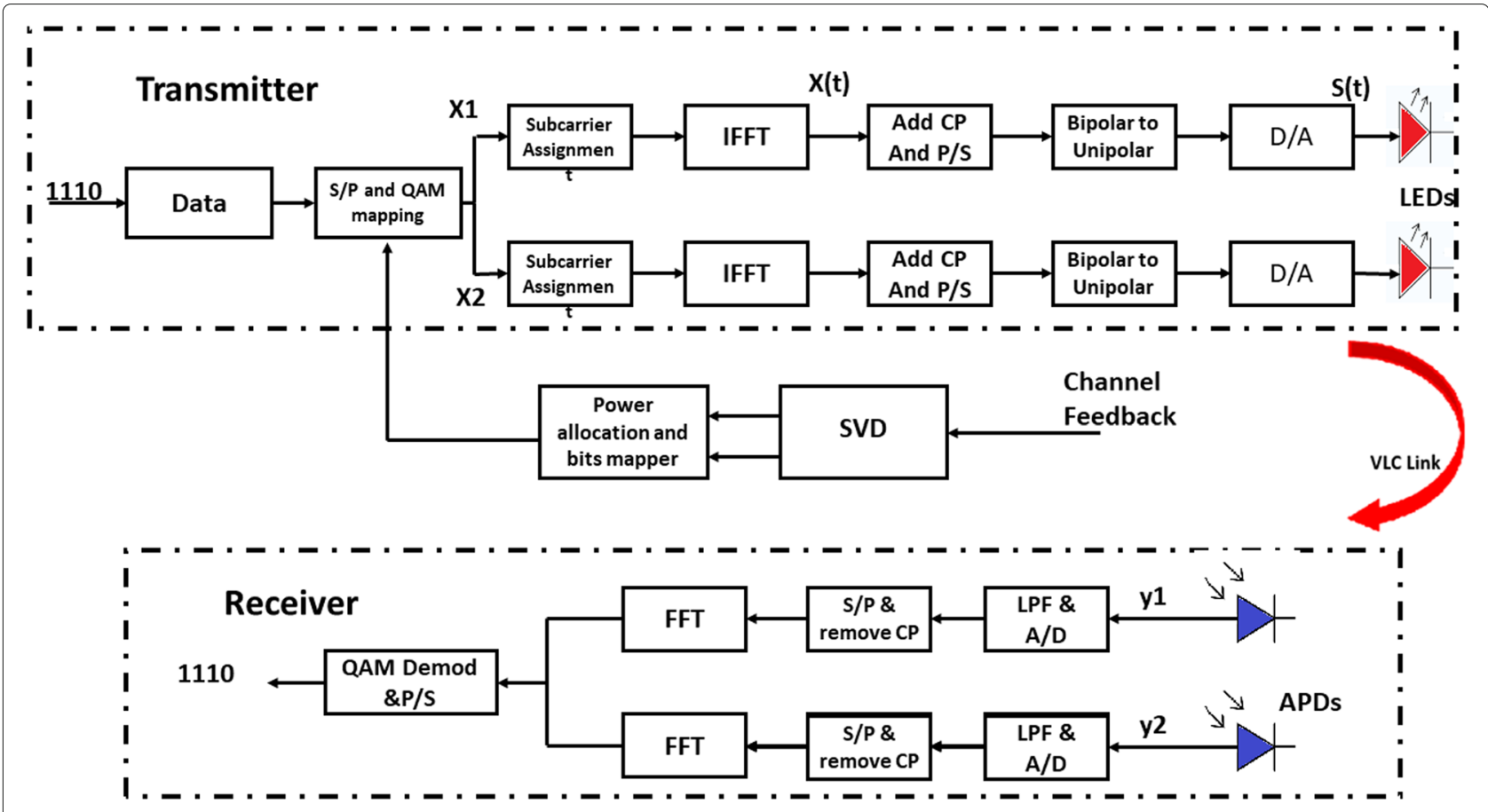

Fig. 3 Simplified diagram of the VLC-based V2V OFDM based scheme

In Fig. 3, a serial-to-parallel $(\mathrm{S} / \mathrm{P})$ converter is used to split a binary source of data into two data streams $\mathbf{x}_{1}, \mathbf{x}_{2}$. Both streams are modulated using QAM, and the resulting symbols are assigned to subcarriers. Afterwards, inverse fast Fourier transform (IFFT) is performed and data is converted back to a single stream using a parallel-to-serial $(\mathrm{P} / \mathrm{S})$ converter, after adding a cyclic prefix (CP). Afterwards, the signal is changed to unipolar by using one of the $\mathrm{O}-\mathrm{OFDM}$ schemes. Finally, the data is converted to ana$\log$ signal $s(t)$ by using a digital-to-analog $(\mathrm{D} / \mathrm{A})$ converter along with low pass filter (shaping filter).

\subsection{DCO-OFDM modulation}

In the DCO-OFDM technique, data is assigned to both odd and even subcarriers. Thus, input vector to the IFFT block $\mathbf{x}$ can be given as

$$
\mathbf{x}=\left[\begin{array}{llllllllll}
0 & s_{1} & s_{2} & \ldots & s_{N / 2-1} & 0 & s_{N / 2-1}^{*} & \ldots & s_{2}^{*} & s_{1}^{*}
\end{array}\right]
$$

where, $N$ is number of subcarriers, and $*$ is conjugate sign.

A real signal can be obtained by imposing the Hermitian symmetry property,

$$
X_{n}=X_{N-n}^{*} \quad n=0,1,2, \ldots, N
$$

This property implies that only half of the OFDM subcarriers can be utilized to generate the real-time domain signal [29]. Therefore, the DCO-OFDM based VLC systems can exploit only half of the given spectrum. Due to central limit theorem, and by the definition of Fourier transform and Euler's identity, $x(t)$ is a Gaussian random variable with zero mean and variance given as

$$
\begin{aligned}
\sigma_{x}^{2} & =\frac{2}{N^{2}}\left(\sigma_{1}^{2}+\sigma_{2}^{2}+\ldots .+\sigma_{N / 2-1}^{2}\right) \\
& =\frac{2}{N^{2}} \sum_{k=0}^{N / 2-1} \sigma_{k}^{2}
\end{aligned}
$$

where $\sigma_{k}^{2}$ is the variance of the $k$ th subcarrier.

The output signal of D/A is an analog real-time signal which is shown in Fig. 4a. This signal cannot be used to directly modulate the LEDs because of its bipolar nature. A certain bias value is added to this signal to convert it to unipolar and is plotted in Fig. 4b. Afterwards, any remaining negative values are clipped at zero and is plotted in Fig. 4c, which introduces clipping distortion. Note, the added DC value increases the transmitter power requirement, while the clipping introduces clipping noise in both the even and odd subcarriers. Therefore, an optimum bias value has to be decided to keep both the transmitter power and the clipping noise within acceptable limits.

The transmitted signal $s(t)$ is given as

$$
s(t)=x(t)+b_{d c}-e_{c}(t)
$$

where $b_{d c}$ is the bias voltage, and $e_{c}(t)$ is clipping noise with a variance $\sigma_{c_{\mathrm{DCO}}}^{2}$. 


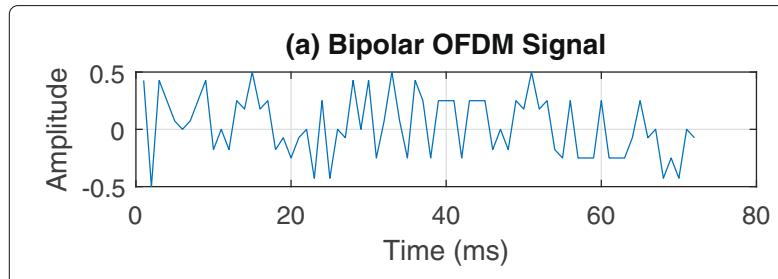

(b) Signal after adding DC bias

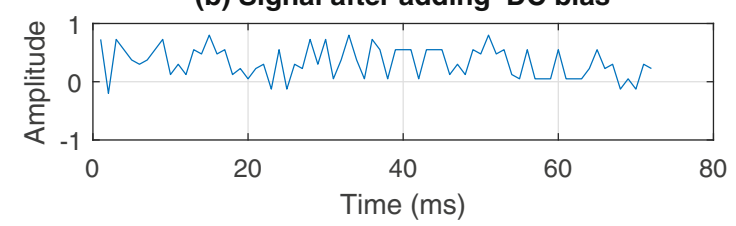

(c)Signal after clipping the remaining negative part

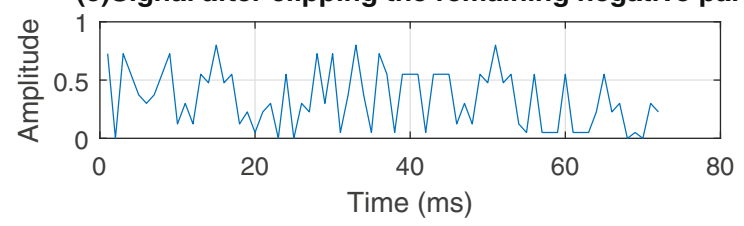

Fig. 4 a OFDM bipolar signal. b DCO-OFDM signal after adding DC bias voltage. c DCO-OFDM signal after clipping the remaining negative

To minimize the clipping noise, the bias voltage $b_{d c}$ should equal to [31]

$$
b_{d c}=2 \sigma_{x} \zeta
$$

where $\zeta$ is the biasing ratio and is given as [31]

$$
\zeta=\frac{b_{d c}}{c_{u}-c_{l}}=\frac{-c_{l}}{c_{u}-c_{l}}
$$

where $c_{u}$ denotes upper clipping level, and $c_{l}$ denotes the lower clipping level.

The variance of the clipping noise $\sigma_{c_{\mathrm{DCO}}}^{2}$ is given as [23]

$$
\begin{aligned}
\sigma_{c_{\mathrm{DCO}}}^{2}= & \sigma_{x}^{2}+\left(b_{d c}^{2}-\sigma_{x}^{2}\right) Q\left(\frac{b_{d c}}{\sigma_{x}}\right)-b_{d c} \sigma_{x} g\left(\frac{b_{d c}}{\sigma_{x}}\right) \\
& -\sigma_{x}^{2} Q\left(\frac{-b_{d c}}{\sigma_{x}}\right)^{2}-\left(\sigma_{x} g\left(\frac{b_{d c}}{\sigma_{x}}\right)-b_{d c} Q\left(\frac{b_{d c}}{\sigma_{x}}\right)\right)^{2}
\end{aligned}
$$

where $g($.$) is the probability density function (pdf) of$ the clipping noise which follows Gaussian distribution and $Q($.$) is the tail probability of the standard Gaussian$ distribution.

For fixed modulation scheme, the maximum channel capacity (bps/Hz) of the DCO-OFDM-VLC system can be given as

$R_{\mathrm{DCO}}=\frac{1}{N+N_{c p}} \sum_{k=1}^{N / 2-1} \log _{2}\left(1+\frac{|H(k)|^{2} \sigma_{k}^{2}}{\sigma_{n}^{2}+|H(k)|^{2} \sigma_{c_{\mathrm{DCO}}}^{2}+\sigma_{\mathrm{ISI}}^{2}}\right)$ where $|H(k)|$ denotes $k$ th channel transfer function, $N_{c p}$ denotes cycling prefix of OFDM, $\sigma_{n}^{2}$ denotes ambient noise power given in (29), and $\sigma_{\text {ISI }}^{2}$ is ISI noise and is given in (30).

\subsection{ACO-OFDM modulation}

In the ACO-OFDM technique, the modulated data is assigned to the odd indexed subcarriers, while the even indexed subcarriers are set to zero. Signals in this scheme are transmitted without any DC-bias.

Referring to Fig. 3, the input vector $\mathbf{x}$ of the IFFT block can be given as

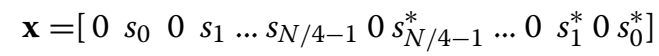

After performing the IFFT, the signal can be written as

$$
x\left[n+\frac{N}{2}\right]=-x[n] \quad n=0,1, \ldots, \frac{N}{2}-1
$$

According to the central limit theorem, and by following Fourier transform definition and Euler's identity, $x(t)$ can be considered a Gaussian random variable with zero mean and variance is given as

$$
\sigma_{x}^{2}=\frac{2}{N^{2}} \sum_{k=1}^{N / 4} \sigma_{2 k-1}^{2}
$$

In the ACO-OFDM scheme, bipolar to unipolar conversion is achieved by removing the negative amplitudes, which will not introduce any distortion to the transmission. However, an LED turn on voltage $V_{\text {tov }}$, has to be added to shift the unipolar signal to the dynamic range of the LED. Hence, any amplitude levels exceeding the upper limit of the LED dynamic range $V_{\max }$ will be clipped, thus resulting in a different type of clipping noise.

The transmitted signal in an ACO-OFDM is written as

$$
s(t)= \begin{cases}x(t) & x(t)>0 \\ 0 & x(t) \leq 0\end{cases}
$$

where $s(t)$ is the clipped signal that has the Gaussian PDF distribution with mean $E[s(t)]=\frac{\sigma_{x}}{\sqrt{2 \pi}}[24]$.

The channel capacity of the fixed modulation scheme for the ACO-OFDM is given as

$$
R_{\mathrm{ACO}}=\frac{1}{N+N_{c p}} \sum_{k=1}^{N / 4} \log _{2}\left(1+\frac{|H(k)|^{2} \sigma_{2 k-1}^{2}}{4 \sigma_{n}^{2}+|H(k)|^{2} \sigma_{c_{\mathrm{ACO}}^{2}}^{2}+\sigma_{\mathrm{ISI}}^{2}}\right)
$$

where $\sigma_{c_{\mathrm{ACO}}}^{2}$ is clipping noise power and is given as [24]

$$
\sigma_{c_{\mathrm{ACO}}}^{2}=\frac{1}{2} \sigma_{x}^{2} Q(\sqrt{2} \eta)\left(1+\eta^{2}\right)-\frac{\sigma_{x}^{2} \eta}{2 \sqrt{\pi}} e^{-\eta^{2}}
$$

Here, $\quad\left(\eta=\frac{I_{\max }-I_{\text {bias }}}{\sigma_{x}}\right)$ is the dimming level, $I_{\max }$ and $I_{\text {bias }}$ are the maximum input current and bias current of the LED, respectively. 
Equation (22) shows the spectral efficiency of the ACO-OFDM is reduced by half as compared to the DCO-OFDM, as only the odd subcarriers are used in transmission.

\section{Noise analysis}

Compared to most previous work, in this work, various noise sources such as the clipping noise from OFDM modulation; quantum (shot) noise from the sunlight (in addition to traditional dark noise and thermal noise at the receiver); and ISI noise are considered.

\subsection{Ambient noise}

At the receiver, the received signal consists of the transmitted signal and the noise signal (comprised of sunlight and other noise sources). We assume that the transmitters and the receivers are on the same horizontal level but the reflectors are not. At the APD, the photocurrent $I_{o}$ due to the received optical power consists of two components, the LOS component and the NLOS component, i.e, $\left(I_{o}=\right.$ $\left.I_{o_{\mathrm{LOS}}}+I_{o_{\mathrm{NLOS}}}\right)$ and is given as

$I_{o}=G A_{R}\left(\int_{\lambda_{1}}^{\lambda_{2}} P_{r}^{\mathrm{LOS}}(\lambda) \gamma(\lambda) T_{o}(\lambda) d \lambda+\int_{\lambda_{1}}^{\lambda_{2}} P_{r}^{\mathrm{NLOS}}(\lambda) \gamma(\lambda) T_{o}(\lambda) d \lambda\right)$

where $\lambda_{1}, \lambda_{2} \in\left[\begin{array}{ll}350 & 750\end{array}\right](\mathrm{nm})$ are the visible light wavelengths, $P_{r}^{\mathrm{LOS}}(\lambda), P_{r}^{\mathrm{NLOS}}(\lambda)$ are given in $(8)$, and (9) respectively for certain wavelength $\lambda, G$ is APD gain, $\gamma(\lambda)$ is the responsivity of the APD given in A/W, and $T_{o}(\lambda)$ is the transmittance of the bandpass optical blue filter.

Similarly, the photocurrent $I_{s}$ produced at APD due to the solar irradiance is given as

$$
I_{s}=G A_{R} \cos \left(\theta_{\text {sun }}\right) \int_{\lambda_{1}}^{\lambda_{2}} P_{s}(\lambda) \gamma(\lambda) T_{o}(\lambda) d \lambda
$$

where $P_{s}(\lambda)$ is the sunlight irradiance given in $W / \mathrm{m}^{2} / \mathrm{nm},\left(\theta_{\text {sun }}\right)$ is the incident angle of the sunlight on the receiver surface as shown in Fig. 2.

The random arrival of the incident photons from both LED light and sunlight result into shot noise. This type of noise can be modeled by Poisson process. Moreover, when the number of incident photons is large, shot noise is approximated by a Gaussian process (using central limit theorem).

According to [25], the shot noise variance is given as

$$
\sigma_{\text {Shot }}^{2}=2 q_{e} G^{2} F\left(I_{o}+I_{s}\right) B
$$

where $q_{e}$ is the electron charge, $F$ is the excess noise; $F=$ $k_{n} G+\left(2-\frac{1}{G}\right)\left(1-k_{n}\right)$ where $k_{n}$ is the holes/electrons ionization rate [34], and $B$ is the APD bandwidth.

\subsection{Other noise sources}

In addition to the shot noise, there are many different types of noises degrading the performance of the VLC system such as dark current noise. Dark current noise is given as

$$
\sigma_{\text {Dark }}^{2}=2 q_{e} G^{2} F I_{d g} B+2 q_{e} I_{d s}
$$

where, $I_{d s}$ is the surface dark current and $I_{d g}$ is the bulk dark current that experiences the avalanche effect of the APD.

Also, due to the random thermal motion of charge carriers, thermal noise is generated, the variance of thermal noise is given as [25]

$$
\sigma_{\text {Thermal }}^{2}=4\left(\frac{K_{B} T_{k}}{R_{L}}\right) F_{n} B
$$

where $K_{B}$ is Boltzmann constant, $T_{k}$ is the temperature in Kelvin, $R_{L}$ is the load resistance given as $50 \Omega$, and $F_{n}$ is the photodiode noise figure.

The total noise variance is given as

$$
\sigma_{n}^{2}=\sigma_{\text {Shot }}^{2}+\sigma_{\text {Dark }}^{2}+\sigma^{2} \text { Thermal }
$$

\subsection{ISI noise}

$I S I_{\text {Noise }}$ with variance $\sigma_{\text {ISI }}^{2}$ is given as

$$
\sigma_{\text {ISI }}^{2}=\gamma^{2}\left(\sum_{k=1}^{N} \int_{T_{d}}^{\infty}\left(h_{k}(t) \otimes s(t)\right) d(t)\right)^{2}
$$

where $T_{d}$ denotes the transmitted optical pulse duration, $\otimes$ is the convolution sign, $\gamma$ denotes the responsitivity of APD, and $k$ is subcarrier index.

The overall noise variance in the proposed system is given as

$$
\sigma_{z}^{2}=\sigma_{n}^{2}+\sigma_{\text {ISI }}^{2}+\sigma_{c}^{2}
$$

\section{Performance analysis and bit loading algorithm}

In this section, performance analysis of the proposed system is presented employing bit loading algorithm. In this model, the DCO-OFDM and the ACO-OFDM schemes are used with both the LOS and the NLOS signal paths. APDs at the receiver converts optical signal to electrical signal with the addition of noise.

\subsection{SNR estimation of subcarrier using SVD-Precoder}

In Fig. 3, received signal with clipping effect before A/D conversion is shown. This received signal can be written as

$$
\left.y_{R}(t)=\sum_{T=1}^{2}\left(\gamma\left(s_{T}(t)+e_{c}(t)\right) \otimes h_{R T}(t)\right)+z_{R}(t)\right)
$$

The matched filter $\mathrm{p}(\mathrm{t})$ is applied to the received signal $y_{R}(t)$ and then its output is sampled at the rate of $T_{s}, y_{R}[n]=y\left(n T_{s}\right)$. 
Let $S_{T}(k)$ is the transmitted signal on the $k$ th subcarrier, and $Y_{R}(k)$ is the received signal. Thereafter, received signal in frequency domain is written as

$$
Y_{R}(k)=\sum_{T=1}^{2}\left(\gamma H_{R T}(k)\left(S_{T}(k)+E_{c}(k)\right)+Z_{R}(k)\right)
$$

where, $Z_{R}(k)$ represents the noise signal in frequency domain, $E_{c}(k)$ is the clipping noise in frequency domain, and $H_{R T}(k)$ is the channel response between Tth LED transmitter and $R$ th receiver on $k$ th subcarrier. In this work, the model described in [10] and [27] is used to evaluate NLOS response.

The estimated channel matrix of $k$ th subcarrier can be expressed as

$$
\mathbf{H}(k)=\left[\begin{array}{ll}
H_{11}(k) & H_{12}(k) \\
H_{21}(k) & H_{22}(k)
\end{array}\right]
$$

\subsubsection{SVD-based pre-coding design}

As RF and VLC have completely different system setups, the optimal structure of user pre-coding matrix is also expected to be different. SVD-based structure is optimal for RF communication systems [32]. However, it is not optimal for VLC communications especially, when a large number of LEDs are used with a requirement of a large biasing voltages.

Nevertheless, since we have assumed small number of LEDs with small bias requirements, SVD technique can be employed to calculate the gain of each sub-carrier. The SVD structure is also practical to use in many aspects. It can distribute the MIMO channel into a set of parallel non-interfering channels [33]. The SVD-precoder scheme solves the channel correlation problem which might cause low spatial multiplexing gain in visible light MIMO communication.

For SVD-precoder, the precoding matrix is given as

$$
\mathbf{H}=\mathbf{U ~ D ~ V}^{*}
$$

where $*$ denotes Hermitian transpose, $\mathbf{V}$ is a $2 \times 2$ unitary matrix, used in transmitter to pre-process transmitted signal, $\mathbf{U}$ is a $2 \times 2$ unitary matrix at the receiver, and $\mathbf{D}$ is a $2 \times$ 2 diagonal matrix with nonnegative diagonal elements (singular values of the matrix $\mathbf{H}$ ).

Therefore, the channels are decomposed into two independent parallel subchannels. The equivalent gain of these two independent subchannels is given as

$$
\mathbf{G}_{o}=\left[\begin{array}{llll}
\lambda_{11} & \lambda_{12} & \ldots & \lambda_{1 k} \\
\lambda_{21} & \lambda_{22} & \ldots & \lambda_{2 k}
\end{array}\right]
$$

The gain of each subcarrier can be found with the matrix $\mathbf{G}_{o}$. Therefore, SNR $[k]$ of $k$ th subcarrier can be calculated as

$$
\operatorname{SNR}[k]=\frac{\lambda_{k}}{\sigma_{z}^{2}(k)}
$$

By considering a target BER (BER $k]$ ) for a certain subcarrier, the modulation order $M_{k}$ can be found using (38) [3]

$$
\operatorname{BER}[k]=\frac{\left(\sqrt{M_{k}}-1\right)}{\sqrt{M_{k}} \log _{2}\left(\sqrt{M_{k}}\right)} \operatorname{erfc}\left(\sqrt{\frac{3 \mathrm{SNR}[k]}{2\left(M_{k}-1\right)}}\right)
$$

\subsection{Adaptive transmission and bit loading scheme}

The bit-loading algorithms is considered to maximize the throughput under total power and spectral mask constraints in OFDM systems. Therefore, subcarriers having high attenuation are re-modulated with lower modulation orders. Similarly, the subcarrier having low attenuation are re-modulated with higher modulation orders. The subcarriers of the O-OFDM signal are modulated by $M-$ $Q A M$; the constellation order $M$ differs from other subcarriers on the basis of SNR. In this work, the algorithm proposed in [28] is utilized, and bits and power to these independent subchannels are adaptively allocated.

The number of bits per symbol of each subcarrier is between 1 and 8 . By obtaining $M_{k}$ for each subcarrier, the transmission rate (bps) for the DCO-OFDM-VLC can be calculated as [6]

$$
R_{\mathrm{DCO}_{\text {Adaptive }}}=\frac{1}{\left(N+N_{c p}\right) T_{s}} \sum_{k=1}^{N / 2-1} \log _{2} M_{k}
$$

where $T_{s}$ is the sampling period.

For the ACO-OFDM-VLC, the transmission rate can be given as [6]

$$
R_{\mathrm{ACO}_{\text {Adaptive }}}=\frac{1}{\left(N+N_{c p}\right) T_{s}} \sum_{k=1}^{N / 4-1} \log _{2} M_{k}
$$

\section{Results and discussions}

\subsection{Simulation environment}

MATLAB $^{\text {TM }}$ software is used to model the proposed system. The parameters and their values used in simulations are summarized in Tables 1, 2, and 3. Multipath propagation with one reflection is considered ${ }^{3}$. All the reflectors surfaces are assumed to have the same reflective factor. Moreover, summer season with the clear sky is considered. It is assumed that the vehicles are moving at same speed in the same direction. The city of Toronto is considered for sunlight intensity calculations, whose location coordinates are given in Table 3. We do Monte Carlo simulation for 5000 iterations, for simulating the proposed VLC channel. To reduce the signal distortion, truncated 'Sinc' pulse filter is used for pulse shaping. White light XM-L LED for the vehicle's headlight 
is considered as a transmitter, due to its enhanced performance for delivering 1040 lumens light with 100 lumens per watt efficacy. Moreover, Hamamatsu-S8664 APD is considered as a receiver. The scheme proposed in [30] is used to model the non-linearity of LED's I-V property. Figure 5 shows that, simulated I-V property fits well with the data obtained from the LED data sheet. Moreover, the linear I-V curve is obtained by linear regression of the data.

\subsection{Bit loading}

The system performance is analyzed by considering various parameters for both types of the O-OFDM schemes. The distributions of bit- and power-loading of the DCOOFDM for 64 subcarriers at $1100 \mathrm{~lx}$ with an $8 \mathrm{~dB}$ and $10 \mathrm{~dB}$ bias is shown in Fig. 6. $128 \mathrm{QAM}$ (7 bits/symbol) is assigned to subcarriers having an SNR of $\geq 14 \mathrm{~dB}$, whereas the BPSK (1 bit/symbol) format is chosen for the subcarriers having SNR $=7 \mathrm{~dB}$ (as seen with $8 \mathrm{~dB}$ bias) and no bits assigned for SNR $<7 \mathrm{~dB}$. Also, Fig. 6 shows clipping noise is decreased with the increase in bias. Therefore, for $10 \mathrm{~dB}$ bias, as the clipping noise is less, the total noise power depend more on channel frequency response rather on $8 \mathrm{~dB}$ bias. As a result of $8 \mathrm{~dB}$ bias, the bit allocation decreases by two bits over 64 subcarriers. Moreover, Fig. 6 shows the power distribution and modulation order of the subcarriers have decreasing trends at high frequency spectrum. Reason for this type of behavior depends upon the low-pass property of the LED. In Fig. 7, bit loading and power distribution for different subcarriers for the ACO-OFDM adaptive modulation scheme is presented, under similar channel conditions as in the DCO-OFDM with a $8 \mathrm{~dB}$ bias and $10 \mathrm{~dB}$ bias respectively. In this technique, only the odd subcarriers are used for data transmission. Power distribution and modulation order of the subcarriers are decreasing with the high frequency spectrum. Maximum of 256 QAM (8 bits/symbol) is assigned to subcarriers having an SNR of about $12 \mathrm{~dB}$, whereas the BPSK ( $1 \mathrm{bit} / \mathrm{symbol})$ format is chosen for the subcarriers having $\mathrm{SNR}=3 \mathrm{~dB}$, and no bits assigned for SNR $<3 \mathrm{~dB}$. A key factor to notice is the number of assigned bits to the ACO-OFDM is higher than the DCO-OFDM scheme. This is can be explained as the DCO-OFDM experiences higher clipping distortion compared to ACO-OFDM at certain illuminance intensity; therefore, a number of loaded bits per subcarrier for the DCO-OFDM is generally lower than in the ACO-OFDM. Also, Fig. 7 shows, increasing the bias decreases the clipping noise. As a result of an $8 \mathrm{~dB}$ bias, the bit allocation decreases by 1 bit over 32 subcarriers.

\subsubsection{Transmission rate}

In Figs. 8 and 9, transmission rates for adaptive DCO-OFDM and ACO-OFDM are shown. The adaptive transmissions are compared with the fixed modulation schemes while maintaining the desired BER at $10^{-5}$. In Fig. 8, it is shown that employing the adaptive transmission in the DCO-OFDM will significantly improve data rate compared to fixed modulations. The clipping distortion will affect the performance of the DCO-OFDM after signal power of $13 \mathrm{dBm}$. In Fig. 9, the transmission rate

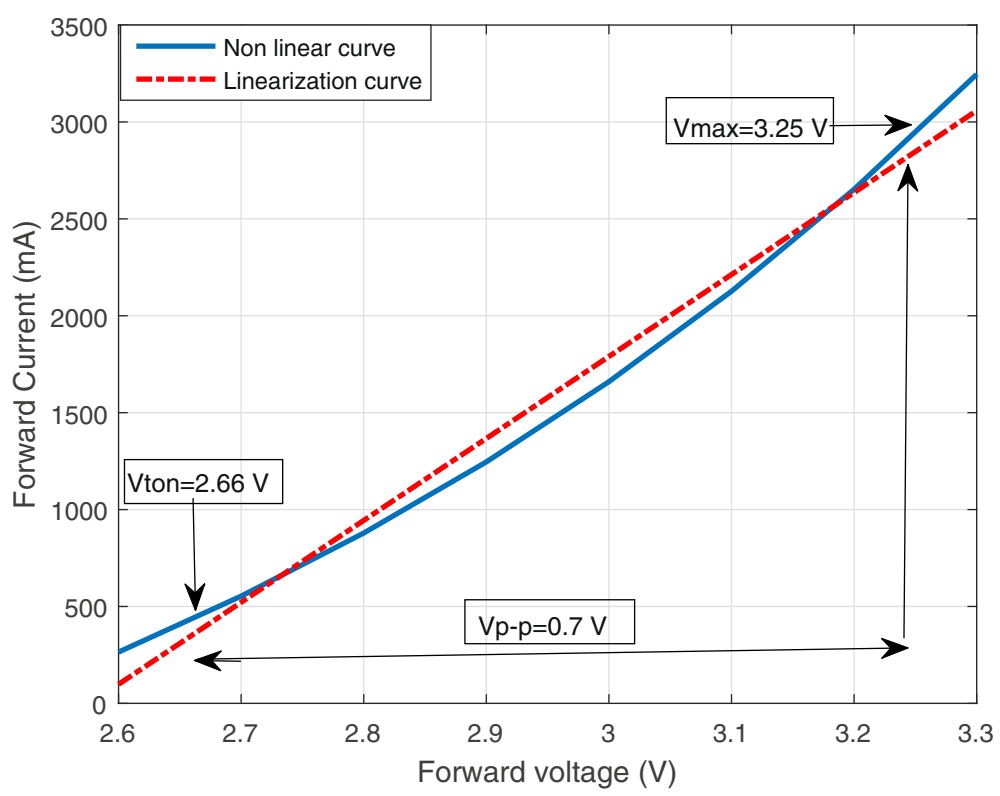

Fig. 5 I-V characteristic curve of the XM-L LED 

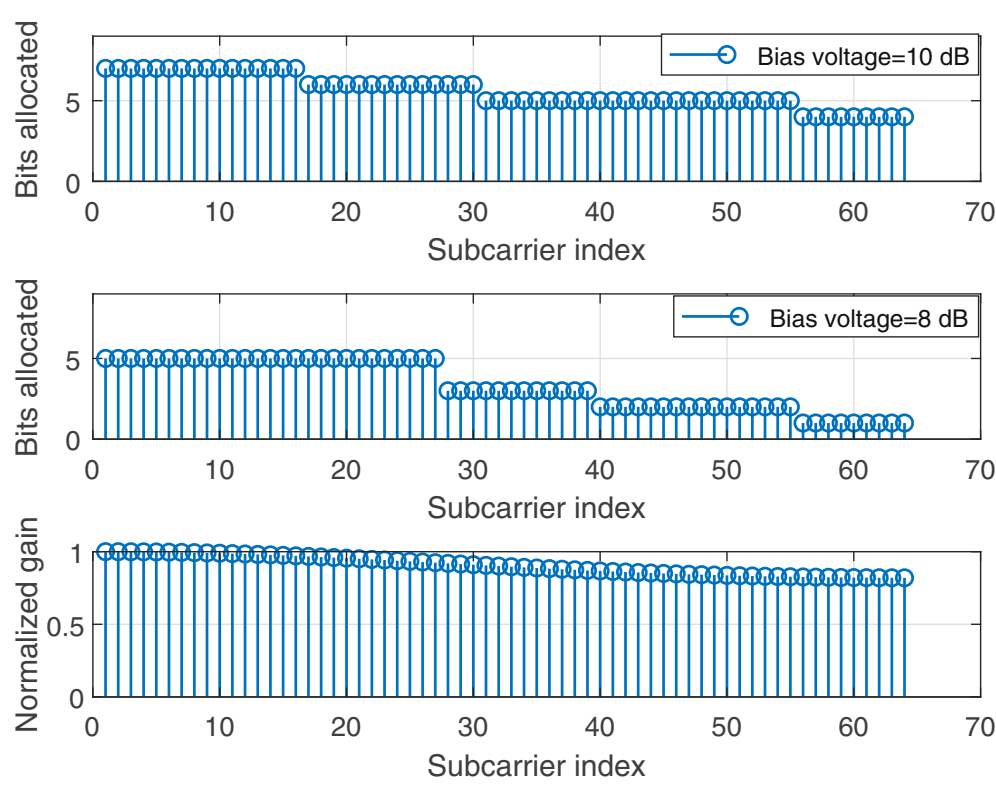

Fig. 6 Number of loaded bits for DCO-OFDM with a $8 \mathrm{~dB}$ bias and $10 \mathrm{~dB}$ bias when $/_{T}\left(\alpha_{T}, \beta_{T}\right)=1100 \mathrm{~lx}$

for the ACO-OFDM is calculated, and it is observed that the clipping distortion degrades the performance when the signal power exceeds $18 \mathrm{dBm}$. Moreover, the maximum achievable rate for the ACO-OFDM is lower than the DCO-OFDM for both types of transmissions schemes; adaptive and fixed. As the ACO-OFDM utilizes dynamic range more effectively with the cost of a halved number of data subcarriers. Figure 10 shows the channel capacity and the achieved spectral efficiency of ACO-OFDM VLC systems with adaptive modulation. For comparison, the performance of the DCO-OFDM scheme is also included. It is observed from that the channel capacity and the achieved spectral efficiency of the ACO-OFDM VLC system are higher than that of the DCO-OFDM VLC system
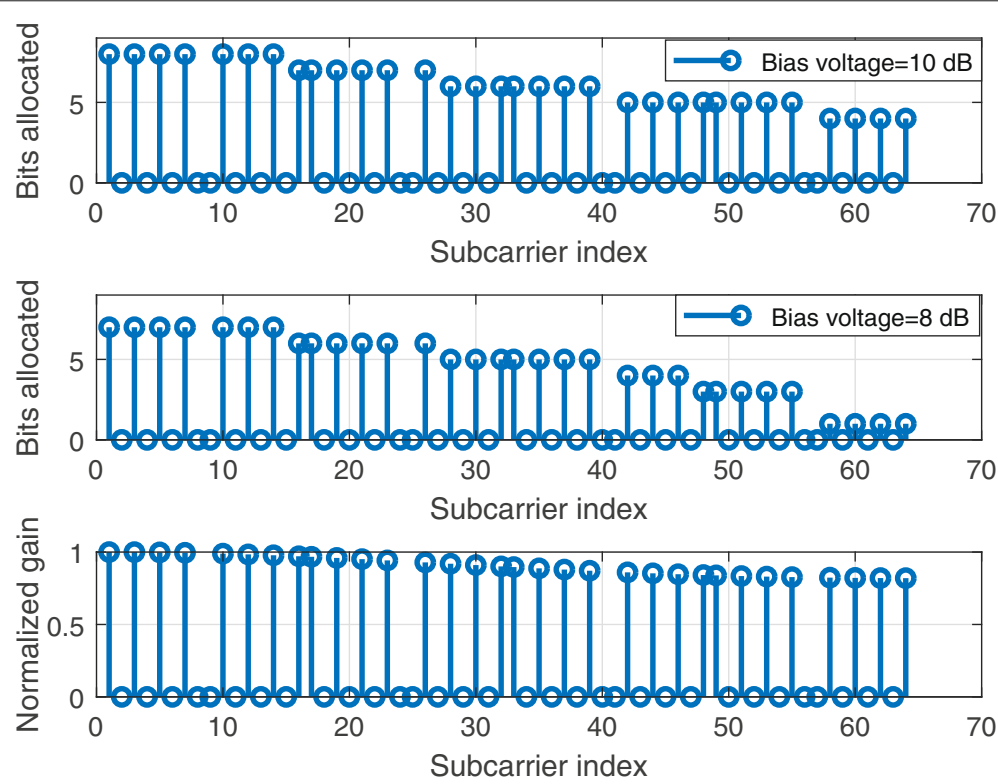

Fig. 7 Number of loaded bits for ACO-OFDM with a $8 \mathrm{~dB}$ bias and $10 \mathrm{~dB}$ bias when $I_{T}\left(\alpha_{T}, \beta_{T}\right)=1100 \mathrm{~lx}$ 


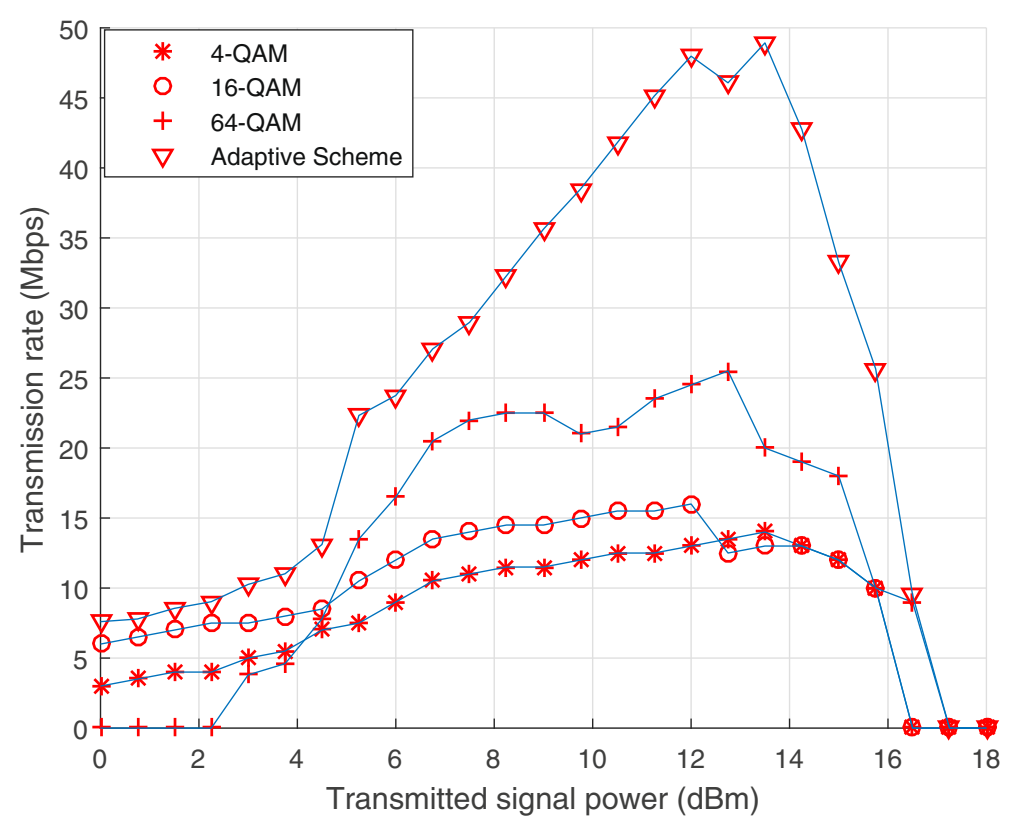

Fig. 8 The transmission rate of DCO-OFDM with adaptive modulation

in the low-SNR region. However, the DCO-OFDM VLC system is superior to the ACO-OFDM VLC system in the high-SNR region.

\subsubsection{BER analysis}

In this section, BER performance for both, the ACOOFDM and the DCO-OFDM, is investigated for adaptive and fixed modulation schemes. Figures 11 and 12 show better SNR performance is achieved for adaptive modulation than fixed modulation. It is also observed that adaptive modulation schemes can meet the target of BER at less SNR value than other QAM schemes. BER performance is not recorded for the SNR range of $(0-3 \mathrm{~dB})$ for the ACO-OFDM, and for the SNR range of $(0-7 \mathrm{~dB})$

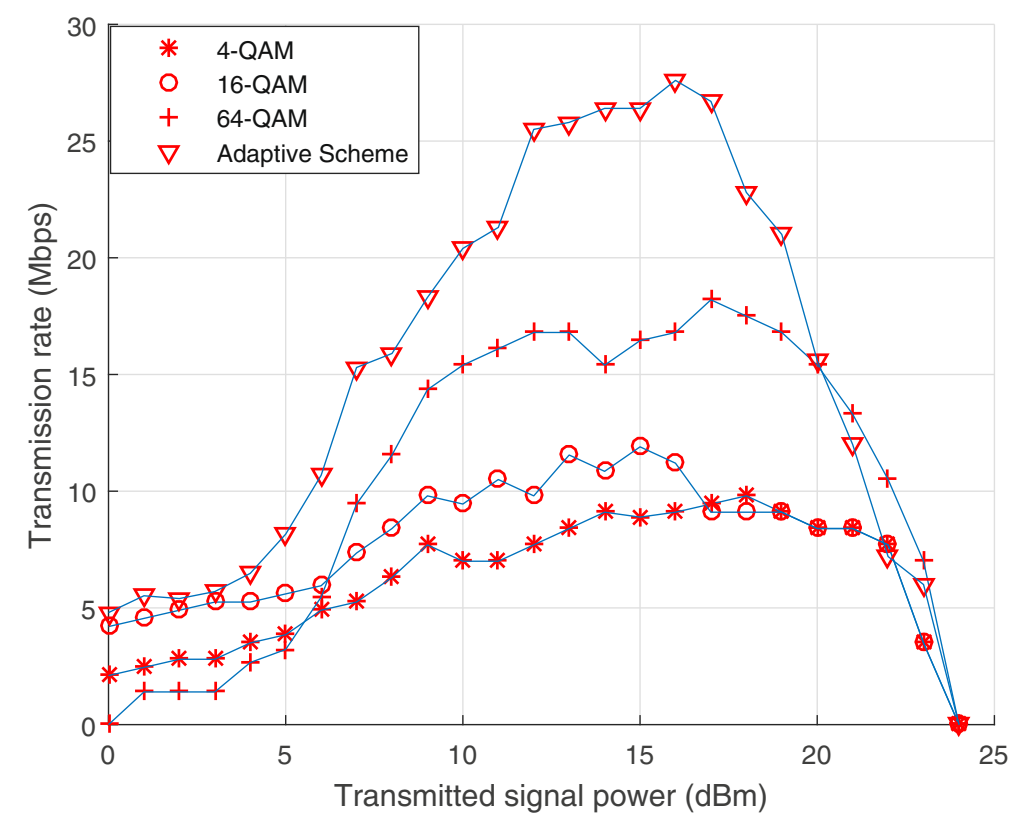

Fig. 9 The transmission rate of ACO-OFDM with adaptive modulation 


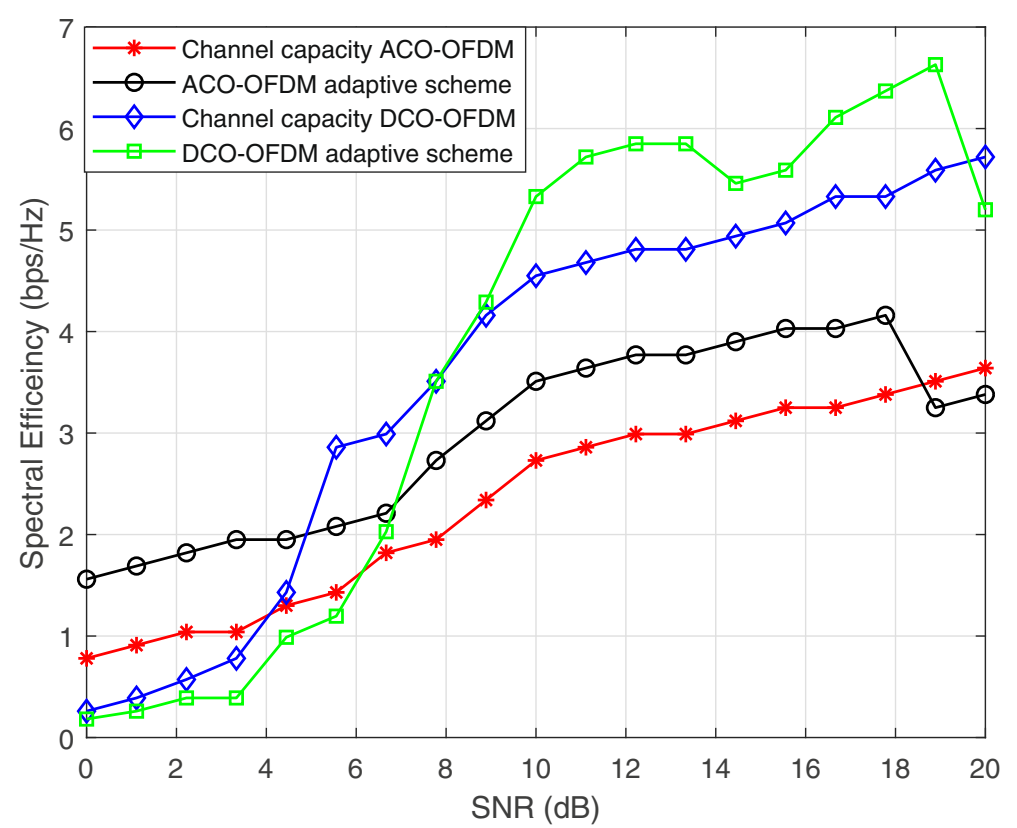

Fig. 10 Spectral efficiency of the two adaptive modulation schemes

for the DCO-OFDM because no bits are transmitted at all between these ranges (according to the bit loading algorithm). The BER performance is not recorded for the SNR $>18 \mathrm{~dB}$ for both schemes because the clipping distortion degrades the performance. Although Fig. 11 shows better performance of ACO-OFDM compared to DCOOFDM, this is due to the inherent characteristics of the DCO-OFDM being sensitive to clipping noise.

\section{Conclusions}

In this paper, a VLC-based V2V system using two types of O-OFDM technique is investigated. Practical channel environment is considered by employing various types of noise and reflectors. A $(2 \times 2)$ MIMO-VLC-based V2V system based on IEEE 802.15.7r1, is considered. An adaptive modulation scheme is used to fully utilize the transmission resources. The proposed model shows

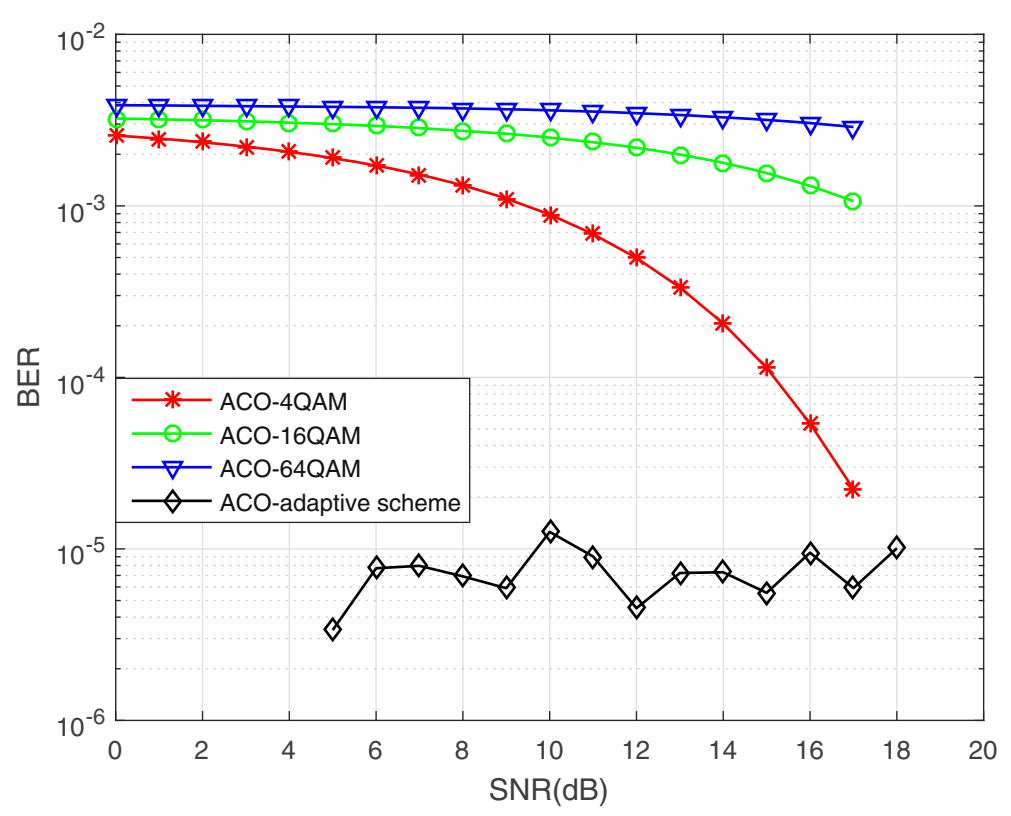

Fig. 11 SNR vs. BER for ACO-OFDM with FFT size $=64$ 


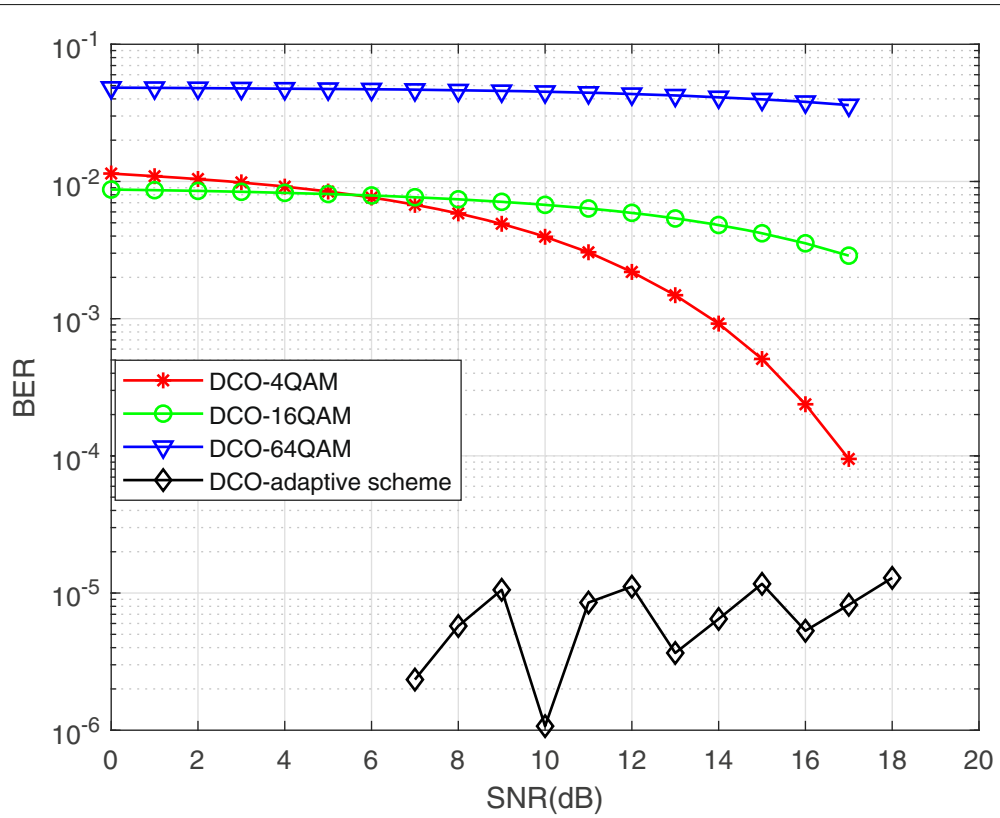

Fig. 12 SNR vs. BER for DCO-OFDM a with FFT size $=64$

performance improvement (along with clipping distortion) for both; DCO-OFDM as well as the ACO-OFDM. The performance comparison between fixed and adaptive modulation schemes is also performed, and superiority in terms of BER is shown for the latter. We showed that a DCO-OFDM adaptive scheme has better data rate performance, while an ACO-OFDM scheme exhibit better BER characteristics. For instance, data rates upto $50 \mathrm{Mbps}$ can be achieved by using the DCO-OFDM scheme, when target bit error rate is $=10^{-5}$.

For future work, the effect of various weather conditions such as rain, and fog on the proposed model will be considered. We intend to use various denoising techniques, such as optical filtering and machine learning algorithms, to improve the performance of a VLC-based V2V system.

\section{Abbreviations \\ ACO-OFDM: Asymmetrically clipped optical orthogonal frequency division multiplexing; APD: Avalanch photo diode; BER: Bit error rate; BW: Bandwidth; CIR: Channel impulse response; DCO-OFDM: Direct current optical orthogonal frequency division multiplexing; FFT: Fast Fourier transform; IFFT: Inverse fast Fourier transform; ISI: Inter symbol interference; ITS: Intelligent transportation system; LED: Light emitting diode; LOS: Line of sight; MIMO: Multiple input multiple output; NLOS: None line of sight; OOK: ON-OFF Keying; OWC: Optical wireless communication; QAM: Quadrature amplitude modulation; RF: Radio frequency; SNR: Signal to noise ratio; SVD: Singular value decomposition; TDE: Time domain equalizer; VLC: Visible light communication; V2I: Vehicle to infrastructure; V2V: Vehicle to vehicle}

\section{Authors' contributions}

The authors have contributed jointly to all parts on the preparation of this manuscript, and all authors read and approved the final manuscript.

\section{Authors' information}

Hasan Farahneh received the B.S. degree from Al-Yarmouk University, Irbid, Jordan in 1985, and the M.Sc. degree from the University of Jordan, Amman, Jordan in 2004. From 2004 to 2013, he was an instructor at Electrical Engineering department at the University of Jordan. In 2013, he joined Ryerson communication lab (RCL). He is currently working toward the Ph.D. degree in the Department of Electrical and Computer Engineering, Ryerson University, Toronto, Canada. His research interest includes wireless and visible light communication. His current research is focused on vehicular communication using visible light spectrum.

Fatima Hussian is adjunct professor at Ryerson university. She is the Editor-in-Chief for IEEE Women in Engineering, Newsletter (Toronto section) and Associate Editor for IEEE Newsletter in Future Directions. Her current research includes Machine Learning, Internet of Things, and Smart Systems with special focus on Intrusion Detection and Resource Allocation. She earned her PhD from Ryerson University in 2015.

Xavier Fernando is a Professor and Director of Ryerson Communications Lab. He was an IEEE Distinguished Lecturer and delivered over 40 invited lectures worldwide. He has (co-)authored over 150 research articles and holds three patents (one pending). He has mono graphed a widely selling book on Radio over Fiber systems. He was a member in the IEEE COMSOC Education Board Working Group on Wireless Communications, 2010-2012. He is the General Chair of IEEE International Humanitarian Technology Conference, 2017. He was the General Chair for IEEE Canadian Conference on Electrical and Computer Engineering (CCECE) 2014. He and his students have won several awards and prizes including the First prize at the Humanitarian Initiatives Workshop of CCECE 2014; Second Prize at the 2014 IHTC Conference in Montreal; Best Paper Award at the International Conference of Smart Grid Engineering (SEGE 2014), UOIT, Canada, 2014; IEEE Microwave Theory and Techniques Society Prize in 2010, Sarnoff Symposium prize in 2009, Opto-Canada best poster prize in 2003 and CCECE best paper prize in 2001. Xavier Fernando earned his PhD from the University of Calgary, Alberta in 2001 in affiliation with TRLabs.

\section{Competing interests}

The authors declare that they have no competing interests.

\section{Publisher's Note}

Springer Nature remains neutral with regard to jurisdictional claims in published maps and institutional affiliations. 
Received: 5 January 2018 Accepted: 25 September 2018 Published online: 19 October 2018

\section{References}

1. P. A. Haigh, F. Bausi, Z. Ghassemlooy, I. Papakonstantinou, H. Le Minh, C. Fléchon, et al., Visible light communications: real time $10 \mathrm{Mb} / \mathrm{s}$ link with a low bandwidth polymer light-emitting diode. Opt. Express. 22(3), 2830-2838 (2014)

2. M. Uysal, Z. Ghassemlooy, A. Bekkali, A. Kadri, H. Menouar, Visible light communication for vehicular networking: Performance study of a V2V system using a measured headlamp beam pattern model. IEEE. Veh. Technol. Mag. 10(4), 45-53 (2015)

3. R. Mesleh, H. Elgala, H. Haas, On the performance of different OFDM based optical wireless communication systems. IEEE/OSA J. Opt. Commun. Netw. 3(8), 620-628 (2011)

4. X. Zhang, Y. Zhou, Y. Yu, P. Han, X. Wang, Comparison and analysis of DCO-OFDM, ACO-OFDM and ADO-OFDM in IM/DD systems. Appl. Mech. Mater. 701-702, 1059-1062 (2014)

5. Q. Wang, Z. Wang, L. Dai, Multiuser MIMO-OFDM for Visible Light Communications. IEEE Photon J. 7(6), 1-11 (2015)

6. L. Wu, Z. Zhang, J. Dang, H. Liu, Adaptive modulation schemes for visible light communications. J. Light. Technol. 33(1), 117-125 (2015)

7. C. Li, Y. Yi, K. Lee, Performance analysis of visible light communication using the STBC-OFDM technique for intelligent transportation systems. Int. J. Electron. 101(8), 1117-1133 (2014)

8. Y. Goto, I. Takai, T. Yamazato, H. Okada, T. Fujii, S. Kawahito, K. Kamakura, A new automotive VLC system using optical communication image sensor. IEEE Photon J. 8(3), 1-17 (2016)

9. P. Luo, Z. Ghassemlooy, H. L. Minh, E. Bentley, A. Burton, X. Tang, Bit-error-rate performance of a car-to-car VLC system using $2 \times 2 \mathrm{MIMO}$. Mediterr. J. Comput. Netw. 11, 400-407 (2015)

10. H. Farahneh, X. Fernando, Channel impulse response characterization of vehicle-to-vehicle communication system in visible light environment. Submitted to Physical communication Journal. (Elsevier, 2017)

11. H. Farahneh, C. Mekhiel, A. Khalifeh, W. Farjow, X. Fernando, in Proc 2016 IEEE Canadian Conference on Electrical and Computer Engineering (CCECE). Shadowing effects on visible light communication channels (Vancouver, BC, 2016), pp. 1-5

12. I. Takai, T. Harada, M. Andoh, K. Yasutomi, K. Kagawa, S. Kawahito, Optical vehicle-to-vehicle communication system using LED transmitter and camera receiver. IEEE Photon J. 6(5), 1-14 (2014)

13. A. Cailean, M. Dimian, Toward environmental-adaptive visible light communications receivers for automotive applications: a review. IEEE Sensors J. 16(9), 2803-2811 (2016)

14. B. Turan, O. Narmanlioglu, S. C. Ergen, M. Uysal, in Proc 2016 IEEE Vehicular Networking Conference (VNC). Broadcasting brake lights with MIMO-OFDM based vehicular VLC, (Columbus, OH, 2016), pp. 1-7

15. M. Beshr, I. Andonovic, M. Hussien, in Proc. SPIE 8540, Unmanned/Unattended Sensors and Sensor Networks IX. The impact of sunlight on the performance of visible light communication systems over the year, (Edinburgh, 2012)

16. I. E. Lee, M. L. Sim, F. W. L. Kung, Performance enhancement of outdoor visible-light communication system using selective combining receiver IET Optoelectron. 3(1), 30-39 (2009)

17. M. S. Islim, H. Haas, An investigation of the solar irradiance effect on visible light communications, in the proceeding of IEEE 28th Annual International Symposium on Personal, Indoor, and Mobil Radio Communications (PIMRC 2017), pp.1-6

18. H. Farahneh, A. Khalifeh, X. Fernando, Photonics North (PN). An outdoor multi path channel model for vehicular visible light communication systems, vol. 2016, (Quebec City, QC, 2016), pp. 1-1

19. B. Schoettle, M. Sivak, M. J. Flannagan, W. J. Kosmatka, Report No.UMTRI-2004-23 the, University of Michigan Transportation Research InstituteA, market-weighted description of low-beam headlighting patterns in the US, (Ann Arbor, 2004)

20. https://eng.libretexts.org/Textbook_Maps/Chemical_Engineering/ Supplemental_Modules_(Materials_Science)/Optical_Properties/ Metallic_Reflection

21. https://en.wikipedia.org/wiki/Headlamp, 2014

22. J. Pan, Q. Chen, W. Qian, L. Geng, Results of a new polarimetric BRDF simulation of metallic surfaces. Infrared Phys Technol. 72, 58-67 (2015)
23. X. Ling, J. Wang, X. Liang, Z. Ding, C. Zhao, Offset and power optimization for DCO-OFDM in visible light communication systems. IEEE Trans. Sig. Process. 64(2), 349-363 (2016)

24. Q. F. Lu, X. S. Ji, K. Z. Huang, 2014 4th IEEE International Conference on Information Science and Technology. Clipping distortion analysis and optimal power allocation for ACO-OFDM based visible light communication, (Shenzhen, 2014), pp. 320-323

25. T. Komine, M. Nakagawa, Fundamental analysis for visible-light communication system using LED lights. Consumer Electronics. IEEE Transactions on. 50(1), 100-107 (2004)

26. Avalench photodiode charactaristics. https://www.hamamatsu.com/ resources/pdf/ssd/s12858_series_etc_kmpd1175e.pdf

27. $H$. Schulze, Frequency-domain simulation of the indoor wireless optical communication channel. IEEE Trans Commun. 64(6), 551-2562 (2016)

28. P. S. Chow, J. M. Cioffi, J. A. C. Bingham, A practical discrete multitone transceiver loading algorithm for data transmission over spectrally shaped channels. IEEE Trans. Commun. 43(234), 773-775 (1995)

29. N. Fernando, Y. Hong, E. Viterbo, Flip-OFDM for unipolar communication systems. IEEE Trans. Commun. 60(12), 3726-3733 (2012)

30. H. Elgala, R. Mesleh, H. Haas, An LED model for intensity-modulated optical communication systems. IEEE Photon. Technol. Lett. 22(11), 835-837 (2010)

31. Z. Yu, R. J. Baxley, G. T. Zhou, EVM and achievable data rate analysis of clipped OFDM signals in visible light communication. EURASIP J Wire Commun Netw. 2012, 321-345 (2012)

32. D. P. Palomar, J. M. Cioffi, M. A. Lagunas, Joint tx-rx beamforming design for multicarrier MIMO channels: A unified framework for convex optimization. Sig Process IEEE Trans. 51(9), 2381-2401 (2003)

33. R. Wang, Q. Gao, J. You, E. Liu, P. Wang, Z. Xu, Y. Hua, Linear transceiver designs for $\mathrm{MIMO}$ indoor visible light communications under lighting constraints. IEEE Trans. Commun. 65(6), 2494-2508

34. G. Keiser, Optical communications essentials. (McGraw-Hill, New York, 2003)

35. X. N. Fernando, B. Balakanthan, Adaptive denoising and equalization of an infrared wireless CDMA system. EURASIP J Wirel Commun Netw, special issue on Optical Wireless Communications. 2005:1, 20-29 (2005)

36. S. Krishnan, X. N. Fernando, K. H. Sun, Non-stationary noise cancellation in infrared wireless receivers Proceedings of the Canadian Conference on Electrical and Computer Engineering (CCECE'2003), (Montreal, Canada, 2003)

37. H. Farahneh, S. Kamruzzaman, X. Fernando, Differential receiver as a denoising scheme to improve the performance of V 2 V-VLC systems proceedings of IEEE International Conference on Communications (ICC 2018), (Kansas City, MO, USA, 2018), pp. 20-24

\section{Submit your manuscript to a SpringerOpen ${ }^{\odot}$ journal and benefit from:}

- Convenient online submission

- Rigorous peer review

- Open access: articles freely available online

- High visibility within the field

- Retaining the copyright to your article

Submit your next manuscript at springeropen.com 